Bull. Austral. Math. Soc.

VOL. 59 (1999) [1-20]

\title{
DYNAMICS OF HOMEOMORPHISMS ON MINIMAL SETS GENERATED BY TRIANGULAR MAPPINGS
}

\author{
Gian Luigi Forti, Luigi Paganoni and Jaroslav Smítal
}

The main goal of the paper is the construction of a triangular mapping $F$ of the square with zero topological entropy, possessing a minimal set $M$ such that $\left.F\right|_{M}$ is a strongly chaotic homeomorphism, as well as other properties that are impossible for continuous maps on an interval.

To do this we define a parametric class of triangular maps on $Q \times I$, where $Q$ is an infinite minimal set on the interval, which are extendable to continuous triangular maps $F: I^{2} \rightarrow I^{2}$. This class can be used to create other examples.

\section{INTRODUCTION}

Let $I=[0,1]$ be the closed unit interval. Let $\mathcal{C}$ denote the class of continuous maps $f: I \rightarrow I$, and $\Delta$ the class of triangular maps $F: I^{2} \rightarrow I^{2}$, that is, the continuous functions defined by

$$
F(x, y)=(f(x), g(x, y))=\left(f(x), g_{x}(y)\right)
$$

The map $f \in \mathcal{C}$ is the base for $F$, and $g_{x}: I \rightarrow I$ is a family of continuous maps depending continuously on $x$. Note that $F$ transforms the layer $I_{x}:=\{x\} \times I$ into the layer $I_{f(x)}$.

Triangular maps have much simpler dynamics than continuous maps of the square in general [7]. This is because the projection $\pi_{1}:(x, y) \mapsto x$ semiconjugates any $F \in \Delta$ to its base $f$ via $f \circ \pi_{1}=\pi_{1} \circ F$. This implies, for example, that Sharkovsky's theorem on the coexistence of periodic orbits remains valid in $\Delta[6]$. Moreover, the projection $\pi_{1}$ maps the class $\operatorname{Per}(F)$ of periodic points of $F$ onto $\operatorname{Per}(f)$, or the class $U R(F)$ of uniformly recurrent points of $F$ onto $U R(f)$. However there are exceptions: homoclinic orbits [7] or isochronically recurrent points [4] of $F$ are not mapped by $\pi_{1}$ onto the corresponding classes of $f$.

Received 23rd March, 1998

Much of the work of this paper was done while the third author was a Visiting Professor at the University of Milano. This research was supported in part by Italian C.N.R., and by the Grant Agency of the Czech Republic, Grant No 201/94/1088. Support of these institutions is gratefully acknowledged.

Copyright Clearance Centre, Inc. Serial-fee code: 0004-9729/99 \$A2.00+0.00. 
A big difference between the dynamics of maps in $\mathcal{C}$ and in $\Delta$ already appears in the simplest cases in which every periodic point of $F$ is a fixed point and the base is linear, see $[5,8]$ (see also [7, Theorem 3]).

However, the class of maps in $\Delta$ of type $2^{\infty}$ (with respect to the Sharkovsky's ordering) is more interesting. There are, for example, maps in $\Delta$ of type $2^{\infty}$ with positive topological entropy [7] but with recurrent points which are not uniformly recurrent [5]. Such maps are impossible in $\mathcal{C}$. In both of the preceeding examples, the map $F$ has a base $f$ of type $2^{\infty}$ with an infinite minimal set $Q$ such that $F$ has "bad" behaviour on the set $\pi_{1}^{-1}(Q)=Q \times I$. (Recall that a set $M$ is a minimal set for a map if it is non-empty, closed and invariant and if no proper subset of $\dot{M}$ has the same properties.)

In the present paper we show that maps of type $2^{\infty}$ in $\Delta$, even homeomorphisms on minimal sets, may have very complicated dynamics. Note that if $M$ is a minimal set for $F$ in $\Delta$, then $\pi_{1}(M)$ is a minimal set for $f$ (this is true for any general semi-conjugacy, see [11]), hence $\pi_{1}(M)$ is either a periodic orbit or a solenoid, that is, a Cantor-type set [1]. The first case, however, implies that $M$ is essentially one-dimensional, so non trivial behaviour is possible only of $\pi_{1}(M)$ is infinite. We shall consider only this case.

In Section 2, starting from a Cantor-type set $Q$ and a map $f: Q \rightarrow Q$ of type $2^{\infty}$, we define a family $\mathcal{T}$ of functions $F$ of type $2^{\infty}$, non-decreasing on any layer and such that $F(Q \times I) \subset Q \times I$. It is always possible to extend each $F \in \mathcal{T}$ to a function $\widetilde{F} \in \Delta$ preserving its type $2^{\infty}$ and the monotonicity on each layer. All these functions have zero topological entropy. Then we define a parametric family $\mathcal{T}_{0} \subset \mathcal{T}$. This construction is based on an idea from [5] and can be further modified to get more general maps.

In Section 3, we construct a subclass $\mathcal{T}_{01}$ of $\mathcal{T}_{0}$ and prove that the maps in this class have a minimal set containing an interval. (The existence of such maps was already proved in [5].)

In Section 4 we show that there are maps in $\mathcal{T}_{01}$ which are distributionally chaotic, and hence, chaotic in the sense of $\mathrm{Li}$ and Yorke on a minimal set. Recall that no map in $\mathcal{C}$ having zero topological entropy can be chaotic on a minimal set [3].

In Section 5 we prove some results concerning functions in $\mathcal{T}_{01}$ and in other classes $\mathcal{T}_{02} \subset \mathcal{T}_{0}$ and $\mathcal{T}_{1} \subset \mathcal{T}$. These results show properties which are impossible in $\mathcal{C}$.

\section{A PARAMETRIC CLASS OF TRIANGULAR MAPS}

Let $\{0,1\}^{\mathbb{N}}$ be the space of all sequences of two symbols equipped with the following metric $\rho: \rho(\underline{\alpha}, \underline{\beta}):=\max \{1 / i: \alpha(i) \neq \beta(i)\}$ for any distinct $\underline{\alpha}=\{\alpha(i)\}_{i \geqslant 1}$ and $\underline{\beta}=$ $\{\beta(i)\}_{i \geqslant 1}$ in $\{0,1\}^{\mathbb{N}}$. Since, as is well known, any Cantor-type set $Q$ is homeomorphic to $\{0,1\}^{\mathbf{N}}$, we may identify an element $x \in Q$ with the corresponding sequence $\underline{x}=$ $x(1) x(2) \cdots$. 
Consider now the function $f: Q \rightarrow Q$ acting on $Q$ as an adding machine, that is, for $\underline{\alpha} \in\{0,1\}^{N}, f(\underline{\alpha})=\underline{\alpha}+1000 \cdots$ where the adding is in base 2 from the left to right; for example, $f(101100 \cdots)=011100 \cdots, f(11100 \cdots)=00010 \cdots$, and so on. Given a point $\underline{x} \in Q$, the point $f^{s}(\underline{x}) \in Q$ is represented by the sequence $\underline{x}_{s}$ obtained by adding (in base 2 ) the sequence $\underline{x}$ and the eventually zero sequence representing the number $s$ written in base 2 from left to right. It is easy to see that $\omega_{f}(\underline{x})=Q$ for any $\underline{x} \in Q$.

Denote by $\mathcal{T}$ the class of maps $F: Q \times I \rightarrow Q \times I$, where $Q$ is a Cantortype set and $F(\underline{x}, y)=(f(\underline{x}), g(\underline{x}, y))$ where $f: Q \rightarrow Q$ is the adding machine, and $g(\underline{x}, \cdot): I \rightarrow I$ is continuous and non-decreasing for any $\underline{x} \in Q$, and the family $g(\underline{x}, \cdot)$ depends continuously on $\underline{x}$ with respect to the uniform metric. Thus $F$ is continuous on $Q \times I$.

Note that each map $F \in \mathcal{T}$ (and obviously also its monotonic extension $\tilde{F} \in \Delta$ ) has topologically entropy $h(F)=0$. Indeed, we have (see [7]).

$$
\sup \left\{h\left(F, I_{x}\right) ; x \in Q\right\}+h(f) \geqslant h(F)
$$

where $h\left(F, I_{x}\right)$ denotes the topological entropy of the map $F: Q \times I \rightarrow Q \times I$ with respect to the compact subset $I_{\underline{x}}$, that is, the entropy $h\left(F, I_{\underline{x}}\right)$ is computed only for trajectories starting from $I_{\underline{x}}$. But since $F^{i}$ is monotonic on $I_{\underline{x}}$ for any $i$, we have clearly $h\left(F, I_{x}\right)=0$, and of course $h(f)=0$ since $f$ is of type $2^{\infty}$. Thus, $h(F)=0$.

Now we describe the construction of the mappings of a special subclass $\mathcal{T}_{0}$ of $\mathcal{T}$.

First we take an increasing sequence of non-negative integers $\left\{k_{i}\right\}_{i=0}^{\infty}$ with $k_{0}=0$ and such that, for all $i \geqslant 1, k_{i}-k_{i-1}-1=: m_{i} \geqslant 1$. Thus $k_{n}=k_{n-1}+m_{n}+1=$ $m_{1}+\cdots+m_{n}+n$. For any $\underline{x} \in Q$, the digits $x\left(k_{1}\right), x\left(k_{2}\right), \ldots$ are called control digits of $\underline{x}$. If

$$
\underline{x}=\underbrace{x(1) \cdots x\left(k_{1}-1\right)}_{m_{1}} x\left(k_{1}\right) \cdots x\left(k_{n-1}\right) \underbrace{x\left(k_{n-1}+1\right) \cdots x\left(k_{n}-1\right)}_{m_{n}} x\left(k_{n}\right) \cdots
$$

we define, for every $n \geqslant 1$,

$\chi_{n}(\underline{x}):=\left(x\left(k_{n-1}+1\right), \ldots, x\left(k_{n}-1\right)\right) \in\{0,1\}^{m_{n}} \quad$ and $\quad\left|\chi_{n}(\underline{x})\right|:=\sum_{i=1}^{m_{n}} x\left(k_{n-1}+i\right) 2^{i-1}$.

Then we consider a family $\Gamma_{n}:=\left\{\varphi(n, j), j=0, \ldots, 2^{m_{n}}-1\right\}$ of functions from $I$ into $I$ satisfying the following properties:

each function $\varphi(n, j) \in \Gamma_{n}$ is continuous and non-decreasing :

$$
\varphi^{r}\left(n, 2^{m_{n}}-1\right) \circ \cdots \circ \varphi^{r}(n, 0)=I d \text { for all } r \geqslant 1
$$


where $I d$ denotes the identity map. We call any map of $\Gamma_{n}$ a map of rank $n$. Moreover we assume that

$$
\lim _{n \rightarrow+\infty} \max _{j}\{\|\varphi(n, j)-I d\|\}=0
$$

where $\|\cdot\|$ is the uniform norm.

Finally we define a function $F: Q \times I \rightarrow Q \times I$ as follows. Take an arbitrary point $\underline{x} \in Q$. If the first zero control digit of $\underline{x}$ is $x\left(k_{n}\right)$, then we define

$$
F(\underline{x}, y)=\left(f(\underline{x}), \varphi\left(n,\left|\chi_{n}(\underline{x})\right|\right)(y)\right)
$$

Otherwise, if $\underline{x}$ has no zero control digits, we set

$$
F(\underline{x}, y)=(f(\underline{x}), y)
$$

Note that (1) and (3) guarantee the continuity of $F$ in $Q \times I$.

The class $\mathcal{T}_{0}$ consists of the functions constructed in this way for any possible choice of the parameters $k_{n}$ and of the families $\Gamma_{n}$.

Let $\pi_{2}:(x, y) \mapsto y$ be the projection on the second variable and put $t_{n}:=2^{k_{n}-1}$ for all $n \geqslant 0$. (Note that $t_{0}=1 / 2$.) Given $F \in \mathcal{T}_{0}$, for any $i \geqslant 0$ and any $y_{0} \in I$, define $y_{i}:=\pi_{2}\left[F^{i}\left(\underline{0}, y_{0}\right)\right]$. Then, for any integer $i \geqslant 0$ we have

$$
F^{i}\left(\underline{0}, y_{0}\right)=\left(f^{i}(\underline{0}), y_{i}\right)=\left(f^{i}(\underline{0}), \psi(i)\left(y_{0}\right)\right) \text {, }
$$

where $\psi(0)=I d$ and, if $1 \leqslant i<t_{n}, \psi(i)$ is a composition of maps $\varphi$ of rank not greater than $n$.

For all $0 \leqslant j \leqslant 2^{m_{n+1}}-1$ and $0 \leqslant r<t_{n}$, we have the following relations

$$
\begin{aligned}
\psi\left(2 j t_{n}+r\right) & =\psi(r) \circ \psi\left(2 j t_{n}\right), \\
\psi\left((2 j+1) t_{n}+r\right) & =\psi_{j}^{*}(r) \circ \psi\left(2 j t_{n}\right),
\end{aligned}
$$

where $\psi_{j}^{*}(r)$ is the function obtained from $\psi(r)$ by replacing all maps $\varphi$ of rank $n$ with $\varphi(n+1, j)$.

Indeed,

$$
f^{2 j t_{n}}(\underline{0})=\underbrace{0 \cdots 0}_{k_{n}} \xi(1) \cdots \xi\left(m_{n+1}\right) 0 \cdots, \quad f^{(2 j+1) t_{n}}(\underline{0})=\underbrace{0 \cdots 01}_{k_{n}} \xi(1) \cdots \xi\left(m_{n+1}\right) 0 \cdots
$$

with $\left|\chi_{n+1}\left(f^{2 j t_{n}}(\underline{0})\right)\right|=\left|\chi_{n+1}\left(f^{(2 j+1) t_{n}}(\underline{0})\right)\right|=\left|\left(\xi(1), \ldots, \xi\left(m_{n+1}\right)\right)\right|=j$. This means that after $2 j t_{n}$ iterations, all the first $n$ control digits are zero and so, for the 
next $r$ iterations, we apply the same functions $\varphi$ as when starting from $\underline{0}$. Conversely, after $(2 j+1) t_{n}$ iterations the $n$-th control digit is equal to one and so, during the next $r$ iterations we proceed as in the previous case, but instead of using the functions $\varphi$ of rank $n$, we apply the function $\varphi(n+1, j)$. This is exactly what is written in formulas (4) and (5). Obviously, if $r<t_{n-1}$, the function $\psi(r)$ does not contain any map of rank $n$ and so $\psi_{j}^{*}(r)=\psi(r)$.

Note that from (5) with $r=0$ we obtain

$$
\psi\left(t_{n}\right)=I d, \quad \psi\left((2 j+1) t_{n}\right)=\psi\left(2 j t_{n}\right),
$$

Now we prove some identities concerning the functions in $\mathcal{T}_{0}$.

LEMMA 1. Let $F \in \mathcal{T}_{0}$. For every $i \geqslant 1$ take $n \geqslant 1$ such that $t_{n-1} \leqslant i<t_{n}$ and consider the representation of $i$ in the form

$$
i=\left(2 \alpha_{n-1}+\beta_{n-1}\right) t_{n-1}+\cdots+\left(2 \alpha_{1}+\beta_{1}\right) t_{1}+2 \alpha_{0} t_{0}
$$

with $0 \leqslant \alpha_{s} \leqslant 2^{m_{s+1}}-1, \beta_{s} \in\{0,1\}$ for $0 \leqslant s \leqslant n-1$ and $\beta_{0}=0$. If

$$
\nu(i)=\max \left\{s \leqslant n-1: \beta_{s}=0\right\}
$$

we write

$$
i=\left(2 \alpha_{n-1}+1\right) t_{n-1}+\cdots+\left(2 \alpha_{\nu(i)+1}+1\right) t_{\nu(i)+1}+2 \alpha_{\nu(i)} t_{\nu(i)}+\theta(i)
$$

Then we have

$$
\psi(i)=\psi(\theta(i)) \circ \varphi^{\gamma(i)}\left(n, \alpha_{n-1}\right) \circ \varphi^{2^{k} n-1-(n-1)}\left(n, \alpha_{n-1}-1\right) \circ \cdots \circ \varphi^{2^{k_{n-1}-(n-1)}}(n, 0)
$$

$$
\psi_{u}^{*}(i)=\psi(\theta(i)) \circ \varphi^{\bar{\gamma}(i)}(n+1, u)
$$

where $\gamma(i)=\sum_{j=\nu(i)}^{n-2} \alpha_{j} 2^{k_{j}-j}\left(<2^{k_{n-1}-(n-1)}\right)$ and $\bar{\gamma}(i)=\gamma(i)+\alpha_{n-1} 2^{k_{n-1}-(n-1)}$.

In particular, for all $n \geqslant 2$ and all $j$ with $0 \leqslant j \leqslant 2^{m_{n}}-1$,

$$
\psi\left(2 j t_{n-1}\right)=\varphi^{2^{k_{n-1}-(n-1)}}(n, j-1) \circ \cdots \circ \varphi^{2^{k_{n-1}-(n-1)}}(n, 0) .
$$

Proof: First we prove (7) by induction on $n$. Let $n=1$, that is, $1 \leqslant i<t_{1}$; we have

$$
\psi(i)=\varphi(1, i-1) \circ \cdots \circ \varphi(1,0)
$$

In this case $i=2 \alpha_{0} t_{0}=\alpha_{0}, \nu(i)=0$ and $\theta(i)=\gamma(i)=0$. So (7) is satisfied. 
Assume (7) true for $n$ and consider $n+1$. We have to find the representation of $\psi(i)$ for all $i$ with $t_{n} \leqslant i<t_{n+1}$. Let

$$
i=\left(2 \alpha_{n}+\beta_{n}\right) t_{n}+\cdots+\left(2 \alpha_{1}+\beta_{1}\right) t_{1}+2 \alpha_{0} t_{0}
$$

and assume first $\beta_{n}=0$. Then, $i=2 \alpha_{n} t_{n}+\theta(i)$ and, by (4),

$$
\psi(i)=\psi(\theta(i)) \circ \psi\left(2 \alpha_{n} t_{n}\right)
$$

Since in this case $\gamma(i)=0,(7)$ is proved if we show that

$$
\psi\left(2 \alpha_{n} t_{n}\right)=\varphi^{2^{k_{n}-n}}\left(n+1, \alpha_{n}-1\right) \circ \cdots \circ \varphi^{2^{k_{n}-n}}(n+1,0) .
$$

We prove (10) by induction on $\alpha_{n}$. By the induction hypothesis and the representation

$$
t_{n}-1=\left(2\left(2^{m_{n}}-1\right)+1\right) t_{n-1}+\cdots+2\left(2^{m_{i}}-1\right) t_{0}
$$

we have

$$
\psi\left(t_{n}-1\right)=\varphi^{\gamma\left(t_{n}-1\right)}\left(n, 2^{m_{n}}-1\right) \circ \varphi^{2^{k_{n-1}-(n-1)}}\left(n, 2^{m_{n}}-2\right) \circ \cdots \circ \varphi^{2^{k_{n-1}-(n-1)}}(n, 0)
$$

where $\gamma\left(t_{n}-1\right)=\sum_{j=0}^{n-2}\left(2^{m_{j+1}}-1\right) 2^{k_{j}-j}=2^{k_{n-1}-(n-1)}-1$.

Now, by (5) and (11)

$$
\psi\left(t_{n}+\left(t_{n}-1\right)\right)=\psi_{0}^{*}\left(t_{n}-1\right) \circ \psi(0)=\psi_{0}^{*}\left(t_{n}-1\right)=\varphi^{2^{k_{n}-n}-1}(n+1,0)
$$

Since

$$
f^{2 t_{n}-1}(\underline{0})=\underbrace{1 \cdots 1}_{k_{n}} 0 \cdots
$$

at the next iteration we apply the map $\varphi(n+1,0)$, thus

$$
\varphi\left(2 t_{n}\right)=\varphi^{2^{k_{n}-n}}(n+1,0),
$$

hence (10) is proved for $\alpha_{n}=1$. Assume it is true for $\alpha_{n}=j$. By (6)

$$
\psi\left((2 j+1) t_{n}\right)=\psi\left(2 j t_{n}\right)
$$

and by (5) and the induction hypothesis we have

$$
\begin{aligned}
\psi\left((2 j+1) t_{n}+\right. & \left.t_{n}-1\right)=\psi_{j}^{*}\left(t_{n}-1\right) \circ \psi\left(2 j t_{n}\right) \\
& =\varphi^{2^{k_{n}-n}-1}(n+1, j) \circ \varphi^{2^{k_{n}-n}}(n+1, j-1) \circ \cdots \circ \varphi^{2^{k_{n}-n}}(n+1,0) .
\end{aligned}
$$


Since

$$
f^{2 j t_{n}+2 t_{n}-1}(\underline{0})=\underbrace{1 \cdots 1}_{k_{n}} \xi(1) \cdots \xi\left(m_{n+1}\right) 0 \cdots
$$

with $\left|\chi_{n+1}\left(f^{2 j t_{n}+2 t_{n}-1}(\underline{0})\right)\right|=\left|\left(\xi(1), \ldots, \xi\left(m_{n+1}\right)\right)\right|=j$, at the next iteration we apply the map $\varphi(n+1, j)$, thus obtaining (10) for $\alpha_{n}=j+1$. Hence (10) is completely proved.

Assume now $\beta_{n}=1$, that is,

$$
i=\left(2 \alpha_{n}+1\right) t_{n}+\cdots+\theta(i)=\left(2 \alpha_{n}+1\right) t_{n}+r
$$

and observe that $\theta(i)=\theta(r)$.

By (5) and (10) we obtain

$$
\psi(i)=\psi_{\alpha_{n}}^{*}(r) \circ \psi\left(2 \alpha_{n} t_{n}\right)=\psi_{\alpha_{n}}^{*}(r) \circ \varphi^{2^{k_{n}-n}}\left(n+1, \alpha_{n}-1\right) \circ \cdots \circ \varphi^{2^{k_{n}-n}}(n+1,0)
$$

If $t_{n-1} \leqslant r<t_{n}$, then $\nu(i)=\nu(r)$ and

$$
\psi_{\alpha_{n}}^{*}(r)=\psi(\theta(r)) \circ \varphi^{\overline{\gamma(r)}}\left(n+1, \alpha_{n}\right)=\psi(\theta(i)) \circ \varphi^{\gamma(i)}\left(n+1, \alpha_{n}\right)
$$

since $\bar{\gamma}(r)=\sum_{j=\nu(i)}^{n-1} \alpha_{j} 2^{k_{j}-j}=\gamma(i)$.

If $r<t_{n-1}$, then $\nu(i)=n-1, \alpha_{n-1}=0$ and so $\bar{\gamma}(r)=\gamma(i)=0$; in this case

$$
\psi_{\alpha_{n}}^{*}(r)=\psi(\theta(r))=\psi(\theta(i))
$$

Thus (7) is proved for $n+1$.

\section{Properties of Minimal Sets for maps in $\mathcal{T}$ and $\mathcal{T}_{0}$}

THEOREM 1. No $F \in \mathcal{T}$ can have a minimal set with non-empty interior in $Q \times I$.

Proof: Assume there is a function $F \in \mathcal{T}$ with a minimal set $M$ containing a nonempty open set $G$ of $Q \times I$. We may assume, without loss of generality, $G \subset Q \times(0,1)$.

Since $\pi_{1}(M)$ is minimal for the base map, $\pi_{1}(M)=Q$ and so, for any $\underline{x} \in Q$ the set $M \cap I_{\underline{x}}$ is non-empty. Let $\underline{x}_{0} \in Q$ and $M_{0}:=M \cap I_{\underline{x}_{0}}$; define $y_{0}=\max \{y$ : $\left.\left(\underline{x}_{0}, y\right) \in M\right\}$. By the minimality of $M$ we have $\omega_{F}\left(\underline{x}_{0}, y_{0}\right)=M$, hence there is an integer $n$ such that $\left(\underline{x}_{n}, y_{n}\right):=F^{n}\left(\underline{x}_{0}, y_{0}\right) \in G$. Since $\underline{x}_{0}$ is the unique preimage of $\underline{x}_{n}$ with respect to $f^{n}$ and $F(M)=M$ (see [1]), we have $F^{n}\left(M_{0}\right)=M \cap I_{x_{n}}$. But $\left.F^{n}\right|_{M_{0}}$ is non-decreasing and so $y_{n}=\max \left\{y:\left(\underline{x}_{n}, y\right) \in M\right\}$, contrary to the fact that $\sup \left\{y:\left(\underline{x}_{n}, y\right) \in M\right\}>y_{n}$. 
THEOREM 2. Suppose that $F \in \mathcal{T}_{0}$ has a minimal set $M$ containing the layer $I_{\underline{0}}$. Then $\left.F\right|_{M}$ is a homeomorphism.

Proof: Since $M$ is a compact set and $F$ is continuous, $\left.F\right|_{M}$ is a homeomorphism if and only if it is one-to-one on any set $M_{\underline{x}}=M \cap I_{\underline{x}}, \underline{x} \in Q$. Consider first the case $\underline{x} \in \operatorname{Orb}(\underline{0})$, that is, $\underline{x}=f^{s}(\underline{0})$ for some $s \geqslant 0$ and let $t_{n}>s$. By $(6), \psi\left(t_{n}\right)=I d$ and this implies that at any step $j<t_{n}$ the function $\varphi$ to be applied to is injective on $\pi_{2}\left[F^{j}\left(I_{\underline{0}}\right)\right]$. Thus $F$ is injective on $F^{s}\left(I_{\underline{0}}\right)$, which, by the minimality of $M$, equals $M_{\underline{x}}$. Take now an aribtrary point $\underline{x} \in Q \backslash \operatorname{Orb}(\underline{0})$. If all control digits of $\underline{x}$ are equal to one, then the function to be applied to is the identity. Assume now that the first zero control digit of $\underline{x}$ is $x\left(k_{n}\right)$ and take the neighbourhood $U$ of $\underline{x}$ in $Q$ given by all $\underline{t} \in Q$ with the first $k_{n}$ digits of their representations equal to those of $\underline{x}$, that is, $\underline{t}(i)=\underline{x}(i)$, $1 \leqslant i \leqslant k_{n}$. Thus, for every $\underline{t} \in U$,

$$
F(\underline{t}, y)=\left(f(\underline{t}), \varphi\left(n,\left|\chi_{n}(\underline{t})\right|\right)(y)\right)=\left(f(\underline{t}), \varphi\left(n,\left|\chi_{n}(\underline{x})\right|\right)(y)\right),
$$

that is, the function $\varphi$ to be applied to is the same for all $\underline{t} \in U$.

Let $\underline{t}_{0}$ be the first point in Orb (ㅁ) belonging to $U$, hence

$$
\underline{t}_{0}=f^{r_{0}}(\underline{0})=x(1) \cdots x\left(k_{n}-1\right) 0 \cdots \in U \cap \operatorname{Orb}(\underline{0})
$$

with $\underline{t}_{0}(i)=0$ for $i \geqslant k_{n}$. Every $\underline{t} \in U \cap \operatorname{Orb}(\underline{0})$ is of the form $\underline{t}=f^{r}(\underline{0})$ with $r \geqslant r_{0}$ and so,

$$
f^{r-r_{0}}(\underline{0})=\underbrace{0 \cdots 0}_{k_{n}} \cdots .
$$

Hence, for the first $r_{0}$ iterations we apply the same maps either starting from $\underline{0}$ or from $f^{r-r_{0}}(\underline{0})$. This implies that, for every $y \in I$,

$$
\pi_{2}\left[F^{r_{0}}(\underline{0}, y)\right]=\pi_{2}\left[F^{r_{0}}\left(f^{r-r_{0}}(\underline{0}), y\right)\right] .
$$

Define $J:=\pi_{2}\left[M_{\underline{t}_{0}}\right]=\pi_{2}\left[F^{r_{0}}\left(I_{\underline{0}}\right)\right]$; it follows that

$$
\begin{aligned}
\pi_{2}\left[M_{\underline{t}}\right]=\pi_{2}\left[F^{r_{0}}\left(F^{r-r_{0}}\left(I_{\underline{0}}\right)\right)\right] & =\pi_{2}\left[F^{r_{0}}\left(f^{r-r_{0}}(\underline{0}), \psi\left(r-r_{0}\right)(I)\right)\right] \\
& \subset \pi_{2}\left[F^{r_{0}}\left(f^{r-r_{0}}(\underline{0}), I\right)\right]=\pi_{2}\left[F^{r_{0}}\left(I_{\underline{0}}\right)\right]=J .
\end{aligned}
$$

By the previous argument concernig the points of the orbit of $\underline{0}$, the map $\varphi\left(n,\left|\chi_{n}(\underline{x})\right|\right)$ is injective on $J$. So it is sufficient to show that $\pi_{2}\left[M_{\underline{x}}\right] \subset J$. Since, by the hypothesis, $I_{\underline{0}} \subset M$, the minimality of $M$ implies

$$
M=\overline{\bigcup_{i=0}^{\infty} F^{i}\left(I_{0}\right)}
$$

whence

$$
M_{\underline{x}} \subset \overline{\bigcup\left\{M_{\underline{t}}: \underline{t} \in \operatorname{Orb}(\underline{0}) \cap U\right\}} .
$$

Since $\pi_{2}\left[M_{\underline{t}}\right] \subset J$ for every $\underline{t} \in \operatorname{Orb}(\underline{0}) \cap U$, by (13) the same holds for the set $\pi_{2}\left[M_{\underline{x}}\right]$. 
REMARK. We conjecture that Theorem 2 is still valid for functions $F \in \mathcal{T}$.

Let us denote by $\sigma_{\delta}$ and $\tau_{\delta}$ the following functions depending on the parameter $\delta \in(0,1)$ :

$$
\sigma_{\delta}(t)=(1-\delta) t, \quad \tau_{\delta}(t)=(1-\delta) t+\delta
$$

Now we introduce the subclass $\mathcal{T}_{01}$ of $\mathcal{T}_{0}$ consisting of those functions $F \in \mathcal{T}_{0}$ that satisfy the following additional conditions:

$\forall n \geqslant 1 \quad \exists j_{n}, 0 \leqslant j_{n} \leqslant 2^{m_{n+1}}-2$, such that:

$$
\left\{\left(1-\delta_{2 n+1}\right)^{2^{k_{2 n}-(2 n)}}\right\}, \quad\left\{\left(1-\delta_{2 n}\right)^{2^{k_{2 n-1}-(2 n-1)}}\right\} \text { are sequences dense in }[0,1]
$$

(Of course, by (3), we must have also $\lim _{n \rightarrow \infty} \delta_{n}=0$ ).

REMARK. Note that given the sequence $\left\{k_{n}\right\}$, it is always possible to construct a (decreasing) sequence $\left\{\delta_{n}\right\}$ converging to 0 and satisfying (17).

Now we prove the following

THeOREM 3. Every $F \in \mathcal{T}_{01}$ has a minimal set $M \supset I_{\underline{0}}$.

Proof: Take a point $\left(\underline{0}, y_{0}\right) \in I_{\underline{0}}$. By $(9)$ and $(14)-(17)$ we have

$$
\begin{aligned}
y_{2\left(j_{2 n+1}+1\right) t_{2 n}} & =\varphi^{2^{k_{2 n}-2 n}}\left(2 n+1, j_{2 n+1}\right) \circ \cdots \circ \varphi^{2^{k_{2 n}-2 n}}(2 n+1,0)\left(y_{0}\right) \\
& =\varphi^{2^{k_{2 n}-2 n}}\left(2 n+1, j_{2 n+1}\right)\left(y_{0}\right)=y_{0}\left(1-\delta_{2 n+1}\right)^{2^{k_{2 n}}-2 n}
\end{aligned}
$$

and similarly

$$
y_{2\left(j_{2 n}+1\right) t_{2 n-1}}=\varphi^{2^{k_{2 n-1}-(2 n-1)}}\left(2 n, j_{2 n}\right)\left(y_{0}\right)=1+\left(y_{0}-1\right)\left(1-\delta_{2 n}\right)^{2^{k_{2 n-1}-(2 n-1)}}
$$

By the hypotheses on the sequence $\left\{\delta_{n}\right\}$, we have

$$
\omega_{F}\left(\underline{0}, y_{0}\right) \supset I_{\underline{0}}
$$

Set $M=\omega_{F}(\underline{0}, 0)$ and let $w=(\underline{u}, v) \in M$. Since $F^{i}(w)$ visits any neighbourhood of $I_{\underline{0}}, \omega_{F}(w)$ contains a point from $I_{\underline{0}}$ and consequently, by $(20),(\underline{0}, 0) \in \omega_{F}(w)$. This implies $\omega_{F}(w) \supset M$, that is, $M$ is a minimal set for $F$, containing $I_{\underline{0}}$. 


\section{Distributional Chaos}

We start this section by defining the notion of distributional chaos.

Let $g$ be a map from a metric space $(S, d)$ into itself. For any pair $(x, y)$ of points of $S$ and any positive integer $n$, we define a distribution function $\Phi_{x y}^{n}: \mathbb{R} \rightarrow[0,1]$ by

$$
\Phi_{x y}^{n}(t)=\frac{1}{n} \#\left\{i: 0 \leqslant i<n \text { and } d\left(g^{i}(x), g^{i}(y)\right)<t\right\} .
$$

Obviously $\Phi_{x y}^{n}$ is a left-continuous non-decreasing function, $\Phi_{x y}^{n}(0)=0$ and $\Phi_{x y}^{n}(t)=1$ for all $t$ greater than the maximum of the numbers $d\left(g^{i}(x), g^{i}(y)\right), 0 \leqslant i \leqslant n-1$. Note that for the definition of each $\Phi_{x y}^{n}$ we need only to know the first $n$ iterates of $g$.

Having the whole sequence $\left\{\Phi_{x y}^{n}(t)\right\}_{n \geqslant 1}$ we set

$$
\Phi_{x y}(t)=\liminf _{n \rightarrow \infty} \Phi_{x y}^{n}(t), \quad \Phi_{x y}^{*}(t)=\limsup _{n \rightarrow \infty} \Phi_{x y}^{n}(t) .
$$

We shall refer to $\Phi_{x y}$ as the lower and $\Phi_{x y}^{*}$ as the upper functions of $x$ and $y$.

If there is a pair $(x, y)$ of points of $S$ such that $\Phi_{u v}(t)<\Phi_{u v}^{*}(t)$ for all $t$ in some non degenerate interval, then we say that $g$ is distributionally chaotic (see $[\mathbf{9}, \mathbf{1 0}]$ ).

The main result of this section is the following.

THEOREM 4. For every $\varepsilon, 0<\varepsilon<1$, there exists a function $F_{\varepsilon} \in \mathcal{T}_{01}$ such that for $u=(\underline{0}, 0)$ and $v=(\underline{0}, 1)$,

$$
\Phi_{u v}^{*}(t)=1,0<t<1 \text { and } \Phi_{u v}(t) \leqslant \varepsilon, 0<t \leqslant 1-\varepsilon .
$$

Proof: Fix $\varepsilon \in(0,1)$. We construct the function $F_{\varepsilon}$ by choosing $j_{n}=0$ for all $n$ and the functions $\varphi(n, j) \in \Gamma_{n}$, depending on integer parameters $a_{n}, b_{n}$ and $m_{n}$, as follows:

$$
\begin{gathered}
\varphi(2 n-1, j)= \begin{cases}\sigma_{\delta_{2 n-1}}, & 0 \leqslant j<a_{2 n-1} \\
I d, & a_{2 n-1} \leqslant j<b_{2 n-1} \\
\sigma_{\delta_{2 n-1}}^{*}, & b_{2 n-1} \leqslant j<a_{2 n-1}+b_{2 n-1} \\
I d, & a_{2 n-1}+b_{2 n-1} \leqslant j<2^{m_{2 n-1}}\end{cases} \\
\varphi(2 n, j)= \begin{cases}\tau_{\delta_{2 n}}, & 0 \leqslant j<a_{2 n} \\
I d, & a_{2 n} \leqslant j<b_{2 n} \\
\tau_{\delta_{2 n}}^{*}, & b_{2 n} \leqslant j<a_{2 n}+b_{2 n} \\
I d, & a_{2 n}+b_{2 n} \leqslant j<2^{m}\end{cases}
\end{gathered}
$$

where $\sigma_{\delta_{2 n-1}}$ and $\tau_{\delta_{2 n}}$ are the functions defined in (14), $\sigma_{\delta_{2 n-1}}^{*}$ and $\tau_{\delta_{2 n}}^{*}$ are their left-inverses given by

$\sigma_{\delta_{2 n-1}}^{*}(t)=\min \left\{1, t /\left(1-\delta_{2 n-1}\right)\right\}, \quad \tau_{\delta_{2 n}}^{*}(t)=\max \left\{0,\left(t-\delta_{2 n}\right) /\left(1-\delta_{2 n}\right)\right\} \quad t \in[0,1]$, 
and $\left\{\delta_{n}\right\}$ is a sequence satifying (17) with $\lim _{n \rightarrow \infty} \delta_{n}=0$.

In this way we get a function $F_{\varepsilon} \in \mathcal{T}_{01}$. Thus what remains to be chosen are the parameters $a_{n}, b_{n}$ and $m_{n}$.

Before starting with the choice of these parameters we need some properties of $F_{\varepsilon}$. Let $J_{i}:=\pi_{2}\left[F_{\varepsilon}^{i}\left(I_{0}\right)\right]$ and $\lambda_{i}:=\left|J_{i}\right|=|\psi(i)(I)|$.

By applying the functions $\sigma_{\delta}$ or $\tau_{\delta}$ to an interval $J \subset I$, we get

$$
\left|\sigma_{\delta}(J)\right|<|J|, \quad\left|\tau_{\delta}(J)\right|<|J| .
$$

Moreover, for every $j \leqslant 2^{m_{n}}-1$ and $s \leqslant r$,

$$
\left|\varphi^{s}(n, j) \circ \varphi^{r}(n, j-1) \circ \cdots \circ \varphi^{r}(n, 0)(J)\right| \leqslant|J|
$$

since first we apply the functions $\sigma_{\delta}$ or $\tau_{\delta}$ and then their left-inverses for a smaller number of times. Thus, by (7) it is easy to prove by induction that for any interval $J \subset I$

$$
|\psi(i)(J)| \leqslant|J|
$$

For any $j$ with $a_{n+1} \leqslant j \leqslant b_{n+1}-1$ we have $\varphi(n+1, j)=I d$; thus, by (9) we obtain $\psi\left(2 j t_{n}\right)=\psi\left(2 a_{n+1} t_{n}\right)$. Then, if we set

$$
\bar{J}:=J_{2 a_{n+1} t_{n}}=\varphi^{2^{k_{n}-n}}\left(n+1, a_{n+1}-1\right) \circ \cdots \circ \varphi^{2^{k_{n}-n}}(n+1,0)(I),
$$

by the structure of the family $\Gamma_{n+1}$ we have

$$
\bar{J}=\sigma_{\delta_{n+1}}^{a_{n+1} 2^{k_{n}-n}}(I) \quad \text { or } \quad \bar{J}=\tau_{\delta_{n+1}}^{a_{n+1} 2^{k_{n}-n}}(I)
$$

according to whether $n$ is even or odd. By (4), (5), (8) and (21) we have

$$
\begin{aligned}
\lambda_{i}=|\psi(i)(I)| & =\left|\psi(r) \circ \psi\left(2 j t_{n}\right)(I)\right|=\left|\psi(r) \circ \psi\left(2 a_{n+1} t_{n}\right)(I)\right| \\
& =|\psi(r)(\bar{J})| \leqslant|\bar{J}|, \quad i=2 j t_{n}+r, 0 \leqslant r<t_{n} \\
\lambda_{i}=|\psi(i)(I)| & =\left|\psi_{j}^{*}(r) \circ \psi\left(2 j t_{n}\right)(I)\right|=\left|\psi_{j}^{*}(r) \circ \psi\left(2 a_{n+1} t_{n}\right)(I)\right| \\
& =\left|\psi_{j}^{*}(r)(\bar{J})\right|=|\psi(\theta(r))(J)| \leqslant|\bar{J}|, \quad i=(2 j+1) t_{n}+r, 0 \leqslant r<t_{n}
\end{aligned}
$$

This implies that, if $\lambda_{2 a_{n+1} t_{n}}=|\bar{J}|<1 /(n+1)$, then

$$
\lambda_{i} \leqslant \lambda_{2 a_{n+1} t_{n}}<\frac{1}{n+1}, \quad 2 a_{n+1} t_{n} \leqslant i<2 b_{n+1} t_{n} .
$$


Now we have to choose the parameters $a_{n}, b_{n}$ and $m_{n}$. The choice will be made iteratively in order to assure that, for $n \geqslant 1$,

$$
\Phi_{u v}^{2 b_{n} t_{n-1}}(1 / n) \geqslant 1-1 / n \quad \text { and } \quad \Phi_{u v}^{t_{n}}(1-\varepsilon) \leqslant \varepsilon .
$$

The relation $\Phi_{u v}^{2 b_{n} t_{n-1}}(1 / n) \geqslant 1-1 / n$ means that the number of $i$ 's less than $2 b_{n} t_{n-1}$ for which $\lambda_{i}<1 / n$ is "almost the same" as $2 b_{n} t_{n-1}$ while $\Phi_{u v}^{t_{n}}(1-\varepsilon) \leqslant \varepsilon$ means that the number of $i$ 's less than $t_{n}$ for which $\lambda_{i}<1-\varepsilon$ is "small" in respect to $t_{n}$.

Let $n=1$. Take $a_{1}=b_{1}=1<2^{m_{1}}$. Then we have $\lambda_{0}=1, \lambda_{1}=1-\delta_{1}$ and $\lambda_{i}=1$ for all $2 \leqslant i \leqslant 2^{m_{1}}=t_{1}$. So, the first inequality of (24) is trivially satisfied and

$$
\Phi_{u v}^{t_{1}}(1-\varepsilon)=\frac{1}{2^{m_{1}}} \#\left\{i: 0 \leqslant i<2^{m_{1}} \text { and } \lambda_{i}<1-\varepsilon\right\} \leqslant \frac{1}{2^{m_{1}}} .
$$

If we choose $m_{1}$ such that $1 / 2^{m_{1}} \leqslant \varepsilon$, then the second inequality of (24) is satisfied.

Assuming we have determined $a_{r}, b_{r}$ and $m_{r}$ for all $r \leqslant n$, now we choose the parameters $a_{n+1}, b_{n+1}$ and $m_{n+1}$. By (22),

$$
\lambda_{2 a_{n+1} t_{n}}=\left(1-\delta_{n+1}\right)^{a_{n+1} 2^{k_{n}-n}},
$$

so we take $a_{n+1}$ so that $\lambda_{2 a_{n+1} t_{n}}<1 /(n+1)$. Now, by (23),

$$
\#\left\{i: 0 \leqslant i<2 b_{n+1} t_{n} \text { and } \lambda_{i}<1 /(n+1)\right\} \geqslant 2\left(b_{n+1}-a_{n+1}\right) t_{n}
$$

and so we can take $b_{n+1}$ so that

$$
\Phi_{u v}^{2 b_{n+1} t_{n}}\left(\frac{1}{n+1}\right) \geqslant \frac{2\left(b_{n+1}-a_{n+1}\right) t_{n}}{2 b_{n+1} t_{n}} \geqslant 1-\frac{1}{n+1},
$$

that is, the first inequality of (24) is satisfied for $n+1$. Assume $m_{n+1}$ has been chosen with $a_{n+1}+b_{n+1}<2^{m_{n+1}}$ and take $a_{n+1}+b_{n+1} \leqslant j<2^{m_{n+1}}$. Then $\varphi(n+1, j)=I d$. If $i=2 j t_{n}+r$ with $0 \leqslant r<t_{n}$ then, by (4), (9) and the structure of $\Gamma_{n+1}$ we have $\psi(i)=\psi(r) \circ \psi\left(2 j t_{n}\right)=\psi(r)$ and so $\lambda_{i}=\lambda_{r}$. The second inequality in (24) implies

$\#\left\{i: i=2 j t_{n}+r\right.$ and $\left.0 \leqslant r<t_{n}: \lambda_{i}<1-\varepsilon\right\}=\#\left\{r: 0 \leqslant r<t_{n}\right.$ and $\left.\lambda_{r}<1-\varepsilon\right\} \leqslant \varepsilon t_{n}$.

Let now $i=(2 j+1) t_{n}+r$ with $0 \leqslant r<t_{n}$. Again, by (5), (8) (9) and the structure of $\Gamma_{n+1}$ we obtain

$$
\psi(i)=\psi_{j}^{*}(r) \circ \psi\left(2 j t_{n}\right)=\psi_{j}^{*}(r)=\psi(\theta(r))
$$

and so $\lambda_{i}=\lambda_{\theta(r)}$. Note that $\theta(r)$ may assume all values from 0 to $t_{\nu(r)}-1$ where $0 \leqslant \nu(r) \leqslant n-1$. (See the notation of Lemma 1.) 
Now we intend counting the number of indices $r$ with $0 \leqslant r<t_{n}$ for which $\nu(r)=l$. This means counting the indices having in their binary representation 0 at the place $k_{l}, 1$ in the places $k_{p}, l<p<n$ and 0 in all places greater or equal to $k_{n}$. Thus the required number is $t_{n} / 2^{n-1}$. These indices can be collected in $t_{n} / t_{l} 2^{n-l}$ blocks of type $l$ containing the numbers having in their binary representation the same digits in the places greater or equal to $k_{l}$, that is, in blocks of indices of the form

$$
r=\left(2 \alpha_{n-1}+1\right) t_{n-1}+\cdots+\left(2 \alpha_{l+1}+1\right) t_{l+1}+2 \alpha_{l} t_{l}+s \text { with } 0 \leqslant s<t_{l}
$$

with the same $a_{q}, l \leqslant q \leqslant n-1$.

For each block $B$ of type $l$ and any index $i=(2 j+1) t_{n}+r, r \in B$, by (7) and the structure of $\Gamma_{n+1}$, we have $\lambda_{i}=|\psi(i)(I)|=|\psi(s)(I)|=\lambda_{s}$ and, by the second inequality in (24), we get

$$
\#\left\{i: i \in B \text { and } \lambda_{i}<1-\varepsilon\right\}=\#\left\{s: 0 \leqslant s<t_{l} \text { and } \lambda_{s}<1-\varepsilon\right\} \leqslant \varepsilon t_{l} .
$$

Since the number of blocks of type $l$ is $t_{n} / t_{l} 2^{n-l}$ and $0 \leqslant l \leqslant n-1$ we obtain

$$
\#\left\{i: i=(2 j+1) t_{n}+r, 0 \leqslant r<t_{n} \text { and } \lambda_{i}<1-\varepsilon\right\} \leqslant \sum_{l=0}^{n-1} \frac{t_{n}}{t_{l} 2^{n-l}} \varepsilon t_{l}=\varepsilon t_{n} \sum_{l=0}^{n-1} \frac{1}{2^{n-l}} .
$$

From (25) and this inequality we get

$$
\#\left\{i: i=2 j t_{n}+r, 0 \leqslant r<2 t_{n} \text { and } \lambda_{i}<1-\varepsilon\right\} \leqslant \varepsilon t_{n} \sum_{p=0}^{n} \frac{1}{2^{p}}=2 t_{n}\left(1-2^{-(n+1)}\right) \varepsilon .
$$

Since $t_{n+1}=2^{m_{n+1}}\left(2 t_{n}\right)$, we conclude that

$$
\begin{aligned}
& \Phi_{u v}^{t_{n+1}}(1-\varepsilon)=\frac{1}{2^{m_{n+1}}\left(2 t_{n}\right)} \#\left\{i: 0 \leqslant i<2^{m_{n+1}}\left(2 t_{n}\right) \text { and } \lambda_{i}<1-\varepsilon\right\} \\
& \leqslant \frac{2\left(a_{n+1}+b_{n+1}\right) t_{n}+2 \varepsilon t_{n}\left(1-2^{-(n+1)}\right)\left(2^{m_{n+1}}-\left(a_{n+1}+b_{n+1}\right)\right)}{2^{m_{n+1}}\left(2 t_{n}\right)} \\
& =\frac{\left(a_{n+1}+b_{n+1}\right)\left(1-\varepsilon\left(1-2^{-(n+1)}\right)\right)}{2^{m_{n+1}}}+\varepsilon\left(1-2^{-(n+1)}\right)
\end{aligned}
$$

and we choose $m_{n+1}$ so that $\Phi_{u v}^{t_{n+1}}(1-\varepsilon) \leqslant \varepsilon$.

Summarising, by Theorems 2, 3 and 4 we have the following

COROLlary 5. For every $\varepsilon, 0<\varepsilon<1$, there exists a function $F_{\varepsilon} \in \mathcal{T}_{01}$ satisfying the following properties:

(i) $F_{\varepsilon}$ has a minimal set $M \subset Q \times I$ such that $\left.F_{\varepsilon}\right|_{M}$ is a homeomorphism;

(ii) $M$ contains points $u$ and $v$ such that

$$
\Phi_{u v}^{*}(t)=1,0<t<1 \text { and } \Phi_{u v}(t) \leqslant \varepsilon, 0<t \leqslant 1-\varepsilon .
$$

Note that the behaviour described in Corollary 5 is impossible in dimension one. Indeed any $f \in \mathcal{C}$ with $h(f)=0$ is not chaotic (in the sense of Li and Yorke) on any minimal set [3]. 


\section{Other RESUlts}

In this section of the paper we present some other results about the functions in the class $\mathcal{T}_{01}$. Moreover we define other subclasses of $\mathcal{T}$ and we prove some properties of them.

THEOREM 6 . For every function $F$ in $\mathcal{T}_{01}$ no point of the layer $I_{0}$ is isochronically recurrent.

PROOF: We have to prove that for every $y_{0} \in I$ there exists a neighbourhood $U=U_{1} \times U_{2}$ of $\left(\underline{0}, y_{0}\right)$ such that for every positive integer $\nu$, there is an integer $r$ for which $F^{r \nu}\left(\underline{0}, y_{0}\right) \notin U$. The proof is analogous to that in [4].

We distinguish two cases: $y_{0}<1$ and $y_{0}=1$.

Let $y_{0}<1$. We choose $U=I \times U_{2}$ with $\sup U_{2}<1$, and take an integer $p$ such that

$$
1-\frac{1}{p}>\sup U_{2}
$$

Consider the set

$$
A:=\left\{n: n \geqslant 1 \text { and } \pi_{2}\left[F^{2\left(j_{n+1}+1\right) t_{n}}(\underline{0}, 0)\right]>1-\frac{1}{p}\right\} .
$$

By (19), the set $A$ contains infinitely many elements and by the monotonicity of the functions $\varphi(n,$.$) , for every t \in I$ we have

$$
\pi_{2}\left[F^{2\left(j_{n+1}+1\right) t_{n}}(\underline{0}, t)\right] \geqslant \pi_{2}\left[F^{2\left(j_{n+1}+1\right) t_{n}}(\underline{0}, 0)\right]>1-\frac{1}{p}
$$

for any $n \in A$.

Take an integer $\nu$ and let $2^{q}+\sum_{i \geqslant q+1} c_{i} 2^{i}, c_{i} \in\{0,1\}, q \geqslant 0$, be its binary representation. Fix any $n \in A$; since

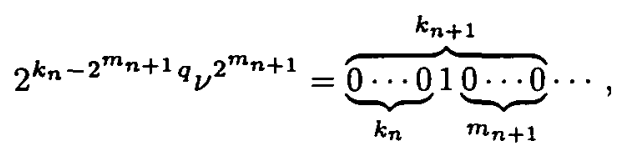

for $r=2\left(j_{n+1}+1\right) t_{n} 2^{k_{n}-2^{m_{n+1}} q} \nu^{2^{m_{n+1}}}-1$ we have

$$
r \nu=\underbrace{0 \cdots 0}_{k_{n}} \xi(1) \cdots \xi\left(m_{n+1}\right) 0 \cdots
$$

with $j_{n+1}+1=\left|\left(\xi(1), \ldots, \xi\left(m_{n+1}\right)\right)\right|$. Since $\mu=r \nu-2\left(j_{n+1}+1\right) t_{n}=\underbrace{0 \cdots 0}_{k_{n+1}} \cdots$, we have $f^{\mu}(\underline{0})=\underbrace{0 \cdots 0}_{k_{n+1}} \cdots$ and $F^{r \nu}\left(\underline{0}, y_{0}\right)=F^{2\left(j_{n+1}+1\right) t_{n}}\left(f^{\mu}(\underline{0}), y_{\mu}\right)$. 
Since $\pi_{2}\left[F^{2\left(j_{n+1}+1\right) t_{n}}\left(f^{\mu}(\underline{0}), t\right)\right]=\pi_{2}\left[F^{2\left(j_{n+1}+1\right) t_{n}}(\underline{0}, t)\right]$ for every $t \in I$, by the definition of $A$ we get

$$
\begin{aligned}
\pi_{2}\left[F^{r \nu}\left(\underline{0}, y_{0}\right)\right] & =\pi_{2}\left[F^{2\left(j_{n+1}+1\right) t_{n}}\left(f^{\mu}(\underline{0}), y_{\mu}\right)\right]=\pi_{2}\left[F^{2\left(j_{n+1}+1\right) t_{n}}\left(\underline{0}, y_{\mu}\right)\right] \\
& \geqslant \pi_{2}\left[F^{2\left(j_{n+1}+1\right) t_{n}}(\underline{0}, 0)\right]>1-\frac{1}{p} .
\end{aligned}
$$

In the case $y_{0}=1$ we proceed in a similar way starting from a neighbourhood $U=I \times U_{2}$ with $\inf \left(U_{2}\right)>0$ and using formula (18).

REMark. The previous result shows that for each function in $\mathcal{T}_{01}$ the point $\underline{0}$ is isochronically recurrent for the base map while it is not the projection of any isochronically recurrent point of the triangular map $F$.

Hence, from Theorems 2, 4 and 6 we get the following

CoRollary 7. For every $\varepsilon, 0<\varepsilon<1$, there exists a function $F_{\varepsilon} \in \mathcal{T}_{01}$ such that:

(i) $F_{\varepsilon}$ has a minimal set $M \supset I_{\underline{0}}$;

(ii) $\left.F_{\varepsilon}\right|_{M}$ is a homeomorphism;

(iii) no point of $I_{0}$ is isochronically recurrent;

(iv) $\Phi_{u v}^{*}(t)=1$ for $0<t<1$ and $\Phi_{u v}(t) \leqslant \varepsilon$ for $0<t \leqslant 1-\varepsilon$, where $u=(\underline{0}, 0)$ and $v=(\underline{0}, 1)$.

Now we define another subclass $\mathcal{T}_{02}$ of $\mathcal{T}_{0}$ as follows: for every $n \geqslant 1$

$$
\begin{aligned}
& \varphi(n, 0)=\varphi(n, 1)=I d, \\
& \varphi^{r}\left(n, 2^{p}-1\right) \circ \cdots \circ \varphi^{r}(n, 0)=I d, 1 \leqslant p \leqslant m_{n}, \text { for all } r \geqslant 1 .
\end{aligned}
$$

THEOREM 8. For every $F \in \mathcal{T}_{02}$ we have

$$
\lim _{s \rightarrow \infty} F^{2^{s}}\left(\underline{0}, y_{0}\right)=\left(\underline{0}, y_{0}\right)
$$

for every $y_{0} \in I$.

Proof: Formulas (6) and (9) and the definition of $\mathcal{T}_{02}$ imply $y_{2^{s}}=y_{0}$ for every integer $s$.

COROLlary 9. There exists $F \in \mathcal{T}_{02}$ such that:

(i) for every $y_{0} \in I, \lim _{s \rightarrow \infty} F^{2^{s}}\left(\underline{0}, y_{0}\right)=\left(\underline{0}, y_{0}\right)$;

(ii) $F$ has a minimal set $M \supset I_{\underline{0}}$;

(iii) $\left.F\right|_{M}$ is a homeomorphism;

(iv) no point of $I_{\underline{0}}$ is isochronically recurrent. 
Proof: By Theorems 2 and 6, the only thing to be proved is that $\mathcal{T}_{01} \cap \mathcal{T}_{02} \neq \emptyset$. It is enough to take in the definition of $\mathcal{T}_{01}, j_{n}=2$ for all $n, \varphi(n, 0)=\varphi(n, 1)=I d$ and choose $\varphi\left(n, j_{n}+1\right)=\varphi(n, 3)$ as the left inverse of $\varphi(n, 2)$. All other functions can be chosen equal to the identity.

REMARK. A one-dimensional map $f$ has zero topological entropy if and only if the set $\left\{x \in I: \lim _{s \rightarrow \infty} f^{2^{s}}(x)=x\right\}$ coincides with the set of the isochronically recurrent points [7, Table 1]. We recall (see Section 2) that our maps have zero topological entropy and so properties (i) and (iv) of Corollary 9 show a completely different behaviour with respect to the one-dimensional case.

To present the last results of the paper we introduce another subclass $\mathcal{T}_{1}$ of $\mathcal{T}$. Let $\left\{k_{i}\right\}_{i=1}^{\infty}$ be an increasing sequence if positive integers with $k_{i}-i \rightarrow+\infty$, and $\left\{\varphi_{i}\right\}_{i=1}^{\infty}$ a sequence of mappings from $I$ into $I$ of the form

$$
\varphi_{i}(t)=t^{s_{i}}, \quad \text { with } s_{i}>0, \lim _{i \rightarrow \infty} s_{i}=1
$$

As in the definition of the class $\mathcal{T}_{0}$, the digits $x\left(k_{1}\right), x\left(k_{2}\right), \ldots$ are called control digits of $\underline{x} \in Q$. We define a function $f: Q \times I \rightarrow Q \times I$ as follows:

If the first zero control digit of $\underline{x}$ is $x\left(k_{n}\right)$,

$$
F(\underline{x}, y)=\left(f(\underline{x}), \varphi_{n}(y)\right)
$$

otherwise $F(\underline{x}, y)=(f(\underline{x}), y)$. The condition $\lim _{i \rightarrow \infty} s_{i}=1$ assures the continuity of $F$. Moreover, it is easy to recognise that $F$ is a homeomorphism of $Q \times I$ onto itself.

THEOREM 10. There exists a function $F \in \mathcal{T}_{1}$ with the following properties:

(i) for any $w \in\{\underline{0}\} \times(0,1)$ we have $\omega_{F}(w)=Q \times I$;

(ii) $F$ has two minimal sets, namely $Q \times\{0\}$ and $Q \times\{1\}$;

(iii) $\{\underline{0}\} \times(0,1) \subset \operatorname{Rec}(F) \backslash U R(F)$;

(iv) for any $u \in\{\underline{0}\} \times(0,1)$ and $v=(\underline{0}, 0)$ or $v=(\underline{0}, 1)$,

$$
\Phi_{u v}^{*}(t)=1, \quad \Phi_{u v}(t)=0, \quad t \in(0,1)
$$

hence $F$ is distributionally chaotic.

Proof: (i) Since the functions $\varphi_{i}$ commute, the value $F^{m}\left(\underline{0}, y_{0}\right)=\left(f^{m}(\underline{0}), y_{m}\right)$ depends only on the number of times any function $\varphi_{i}$ is applied.

Given a positive number $r$, take $n$ so that $k_{n} \leqslant r<k_{n+1}$. Then the points $f^{i}(\underline{0}), 0 \leqslant i<2^{r}$ are represented by all the $2^{r}$ sequences

$$
a_{1}, \cdots a_{r} 0 \cdots, \quad a_{i} \in\{0,1\}, 1 \leqslant i \leqslant r
$$


which have the $(n+1)$-th control digit equal zero and so the only functions that may enter in the expression of $y_{i}, 1 \leqslant i \leqslant 2^{r}$, are $\varphi_{1}, \ldots, \varphi_{n+1}$. The number of times the function $\varphi_{i}, 1 \leqslant i \leqslant n+1$, enters the expression of $y_{2^{r}}$ equals the number of sequences $a_{1} \cdots a_{r} 0 \cdots$ having $a_{k_{i}}=0$ and $a_{k_{s}}=1$ for all $1 \leqslant s<i$. This number is $2^{r-i}$ for $1 \leqslant i \leqslant n$, and $2^{r-n}$ for $i=n+1$. So, we have

$$
y_{2^{k_{n}}}=\varphi_{1}^{2^{k_{n}}-1} \circ \varphi_{2}^{2^{k_{n}-2}} \circ \cdots \circ \varphi_{n-1}^{2^{k_{n}-(n-1)}} \circ \varphi_{n}^{2^{k_{n}-n}} \circ \varphi_{n+1}^{k_{n}-n}\left(y_{0}\right) .
$$

Since

$$
f^{2^{k_{n}}}(\underline{0})=\underbrace{0 \cdots 0}_{k_{n}} 10 \cdots,
$$

for the next $2^{k_{n}}$ iterations we use exactly the same functions as starting from $\underline{0}$. We may proceed in this way until the $k_{n+1}$ digit is zero. Thus, for all $m$ with $2 \leqslant m \leqslant$ $2^{k_{n+1}-k_{n}-1}$ we get

$$
y_{m 2^{k_{n}}}=\varphi_{1}^{m 2^{k_{n}-1}} \circ \varphi_{2}^{m 2^{k_{n}-2}} \circ \cdots \circ \varphi_{n-1}^{m 2^{k_{n}-(n-1)}} \circ \varphi_{n}^{m 2^{k_{n}-n}} \circ \varphi_{n+1}^{m 2^{k_{n}-n}}\left(y_{0}\right) .
$$

In order to construct the function $F$ we start by imposing on the sequence $\left\{s_{i}\right\}$ the additional condition

$$
s_{2 i-1}^{2} s_{2 i}=1, \quad i \geqslant 1
$$

This implies

$$
\varphi_{2 i-1}^{2} \circ \varphi_{2 i}=I d, \quad i \geqslant 1 .
$$

Hence, by (27) and (28) for all $n \geqslant 1$ we obtain

$$
\begin{cases}y_{m 2^{k_{2 n}}}=\varphi_{2 n+1}^{m 2^{k_{2 n}-2 n}}\left(y_{0}\right)=y_{0}^{s_{2 n+1}^{\left(m 2^{k_{2 n}-2 n}\right)}}, & 1 \leqslant m \leqslant 2^{k_{2 n+1}-k_{2 n}-1} \\ y_{m 2^{k_{2 n-1}}}=\varphi_{2 n}^{m 2^{k_{2 n-1}-2 n}}\left(y_{0}\right)=y_{0}^{s_{2 n}^{\left(m 2^{k_{2 n-1}-2 n}\right)}}, & 1 \leqslant m \leqslant 2^{k_{2 n}-k_{2 n-1}-1} .\end{cases}
$$

We want to show that it is possible to choose the sequence of parameters $\left\{s_{n}\right)$ in order to assure that

$$
\forall y_{0} \in(0,1), \quad \omega_{F}\left(\left(\underline{0}, y_{0}\right)\right) \supset I_{\underline{0}} .
$$

Since the $\omega$-limit sets are strongly $F$-invariant, this implies $\omega_{F}\left(\left(\underline{0}, y_{0}\right)\right)=Q \times I$.

To this aim it is enough to assure that the values given by (29) with $m=1$ are dense in $I$ and this is equivalent to requiring that

$$
2^{k_{2 n}-2 n} \log \left(s_{2 n+1}\right) \text { is dense in }(-\infty,+\infty) .
$$


If $\left\{s_{2 n+1}\right\}$ is a sequence satisfying (30) and

$$
\lim _{n \rightarrow+\infty} s_{2 n+1}=1
$$

then the whole sequence $\left\{s_{n}\right\}$ constructed by using (28) satisfies the required property $\lim _{n} s_{n}=1$. To satisfy $(30)$ and (31) we define the sequence $\left\{s_{2 n+1}\right\}_{n=1}^{\infty}$ by

$$
\log \left(s_{2 n+1}\right)=\frac{\sigma_{n}}{2^{k_{2 n}-2 n}}
$$

where $\left\{\sigma_{n}\right\}_{n=1}^{\infty}$ is a sequence dense in $(-\infty,+\infty)$ satisfying

$$
\left|\frac{\sigma_{n}}{2^{k_{2 n}-2 n}}\right|<\frac{1}{n}
$$

So (i) is proved. In the following, given the sequence $\left\{\sigma_{n}\right\}$, we show how to construct the sequence $\left\{k_{n}\right\}$ in order to satisfy (33).

Property (ii) is obvious. By (i), every point $w \in\{\underline{0}\} \times(0,1)$ is recurrent and, by (ii), $\omega_{F}(w)$ is not a minimal set. So $w$ is not uniformly recurrent (see [1]). Now we prove (iv). In order to assure (26) we take sequence $\left\{\sigma_{n}\right\}$, dense in $(-\infty,+\infty)$ and such that $\sigma_{2 n-1}<0$ and $\sigma_{2 n}>0$.

Now we recursively define the sequences $\left\{k_{n}\right\}$ and $\left\{s_{n}\right\}$.

We start the recursive process by taking $k_{1}$ arbitrarily, $s_{1}=s_{2}=1$ and $k_{2}>k_{1}$ satisfying (33). Assume now we have constructed $k_{i}, s_{i}$ for $i \leqslant 2 n$ so that (33) is satisfied. By (28) and (32) we immediately get $s_{2 n+1}$ and $s_{2 n+2}$.

Suppose now $n$ even [ $n$ odd]. We take $0<\rho_{n}<1 / 2 n$ such that, for $y_{0} \leqslant \rho_{n}$ $\left[y_{0} \geqslant 1-\rho_{n}\right]$ and for all $j$ with $0 \leqslant j<2^{k_{2 n}}$, we have

$$
y_{j}<\frac{1}{2 n} \quad\left[y_{j}>1-\frac{1}{2 n}\right]
$$

This is possible since only a finite number of continuous functions enter in the expression of $y_{j}$ and for them both points 0 and 1 are fixed.

Then we find an integer $p$ so that, for $y_{0}=1-(1 / 2 n)\left[y_{0}=(1 / 2 n)\right]$,

$$
y_{p 2^{k_{2 n}}}=\varphi_{2 n+1}^{p 2^{\left(k_{2 n}-2 n\right)}}\left(y_{0}\right)<\rho_{n} \quad\left[y_{p 2^{k_{2 n}}}=\varphi_{2 n+1}^{p 2^{\left(k_{2 n}-2 n\right)}}\left(y_{0}\right)>1-\rho_{n}\right]
$$

Now, we choose $k_{2 n+1}$ so that

$$
\frac{p 2^{k_{2 n}}}{2^{k_{2 n+1-1}}}<\frac{1}{2 n}
$$

and $k_{2 n+2}>k_{2 n+1}$ satisfying (33). 
Now we show that the function $F$ constructed in this way satisfies (26). Take $y_{0} \in(0,1)$ and $n_{0}$ such that $y_{0} \in\left[\left(1 / 2 n_{0}\right), 1-\left(1 / 2 n_{0}\right)\right]$. Choose $n$ even and greater than $n_{0}$. For every $r, p 2^{k_{2 n}} \leqslant r \leqslant 2^{k_{2 n+1}-1}$ we can write $r=m 2^{k_{2 n}}+j$ with $p \leqslant m \leqslant 2^{k_{2 n+1}-k_{2 n}-1}$ and $0 \leqslant j<2^{k_{2 n}}$. So, by (29), (34) and (35)

$$
y_{m 2^{k n}} \leqslant y_{p 2^{k_{2 n}}}<\rho_{n} \text { and } y_{r}<\frac{1}{2 n} \text {. }
$$

Thus

$$
\#\left\{i: 0 \leqslant i<2^{k_{2 n+1}-1} \text { and } y_{i}<\frac{1}{2 n}\right\} \geqslant 2^{k_{2 n+1}-1}-p 2^{k_{2 n}},
$$

and so, by (36)

$$
\Phi_{u v}^{2^{k_{2 n+1}-1}}\left(\frac{1}{2 n}\right) \geqslant 1-\frac{p 2^{k_{2 n}}}{2^{k_{2 n+1}-1}}>1-\frac{1}{2 n} .
$$

Hence we conclude that $\Phi_{u v}^{*}(t)=1$ for $t \in(0,1)$.

Similarly, if we take $n$ odd, we get

$$
\Phi_{u v}^{2^{k} 2 n+1-1}\left(1-\frac{1}{2 n}\right)<\frac{1}{2 n}
$$

and so $\Phi_{u v}(t)=0$ for $t \in(0,1)$.

REMARK. Again the properties proved in Theorem 10 are impossible in $\mathcal{C}$. Indeed for a one-dimensional map $f$ with $h(f)=0$ we have $\operatorname{Rec}(f)=U R(f)$ and each $\omega$-limit set contains only one minimal set. The next theorem shows something more: the existence of a triangular map $F$ with $h(F)=0$ having an $\omega$-limit set containing infinitely many minimal sets.

THEOREM 11. There exists a triangular map $F$ of type $2^{\infty}$, strictly increasing on any layer $I_{x}$, having an $\omega$-limit set containing uncountably many minimal sets.

Proof: By [2, Theorems 6.2,6.5] there exists a function $f \in \mathcal{C}$ of type $2^{\infty}$ having an infinite $\omega$-limit set $\widetilde{Q} \supset Q$ containing isolated points and such that $\widetilde{Q} \backslash Q$ is a single orbit disjoint from $Q$. Moreover, this function acts as the adding machine on $Q$ and for every $x \in \widetilde{Q} \backslash Q$ we have $\omega_{f}(x)=Q$. We take such a function as base of the triangular map we are constructing. We choose $p_{0} \in \widetilde{Q} \backslash Q$ with $\operatorname{Orb}\left(p_{0}\right)=\widetilde{Q} \backslash Q$ and associate to it the zero sequence. Then we code $\operatorname{Orb}\left(p_{0}\right)$ by associating to each point $p_{n}=f^{n}\left(p_{0}\right)$ the corresponding sequence $f^{n}(\underline{0})$. Now we define $\widetilde{F}(x, y)=\left(f(x), g_{x}(y)\right)$ on $\widetilde{Q} \times I$ as follows: for $x \in Q, g_{x}=I d$ and for $x \in \widetilde{Q} \backslash Q, g_{x}$ as in the construction of the class $\mathcal{T}_{1}$ on the corresponding points of Orb $(\underline{0})$. Arguing as in the proof of Theorem 9 we get $\omega_{\tilde{F}}(z)=Q \times I$ for any $z \in(\widetilde{Q} \backslash Q) \times(0.1)$. Clearly, any set $Q \times\{a\}$ is a minimal set for $\widetilde{F}$ contained in $\omega_{\widetilde{F}}(z)$. It is easy to see that it is possible to extend $\widetilde{F}$ continuously to a triangular map $F: I^{2} \rightarrow I^{2}$ increasing on any layer. 


\section{REFERENCES}

[1] L.S. Block and W.A. Coppel, Dynamics in one dimension, Lecture Notes in Math. 1513 (Springer-Verlag, Berlin, Heidelberg, New York, 1992).

[2] A.M. Bruckner and J. Smítal, 'A characterization of $\omega$-limit sets of maps of the interval with zero topological entropy', Ergodic Theory Dynamical Systems 13 (1993), 7-19.

[3] V.V. Fedorenko, A.N. Sharkovsky and J. Smítal, 'Characterizations of weakly chaotic maps of the interval', Proc. Amer. Math. Soc. 110 (1990), 141-148.

[4] G.L. Forti and L. Paganoni, 'On some properties of triangular maps', Grazer Math. Ber. (to appear).

[5] G.L. Forti, L. Paganoni and J. Smítal, 'Strange triangular maps of the square', Bull. Austral. Math. Soc 51 (1995), 395-415.

[6] P.E. Kloeden, 'On Sharkovsky's cycle coexistence ordering', Bull. Austral. Math. Soc. 20 (1979), 171-177.

[7] S.F. Kolyada, 'On dynamics of triangular maps of the square', Ergodic Theory Dynamical Systems 12 (1992), 749-768.

[8] L. Paganoni and P. Santambrogio, 'On some strange properties of triangular maps', Grazer Math. Ber. (to appear).

[9] B. Schweizer and J. Smítal, 'Measures of chaos and a spectral decomposition of dynamical systems on the interval', Trans. Amer. Math. Soc. 344 (1994), 737-753.

[10] B. Schweizer, A. Sklar and J. Smítal, 'Distributional (and other) chaos and its measurement', (preprint).

[11] K.S. Sibirsky, Introduction to topological dynamics (Noordhoff International Publishers, Leiden, 1975).

Dipartimento di Matematica

Università degli Studi di Milano

via C. Saldini 50

20133 Milano

Italy

Institute of Mathematics

Silesian University

74601 Opava

Czech Republuc
Dipartimento di Matematica

Università degli Studi di Milano

via C. Saldini 50

20133 Milano

Italy 